\title{
ON EXPLICIT ESTIMATES FOR LINEAR FORMS \\ IN THE VALUES OF A CLASS OF E-FUNCTIONS \\ Xu Guangshan and Wang LianXiang
}

\begin{abstract}
We apply methods of Mahler to obtain explicit lower bounds for certain combinations of $E$-functions satisfying systems of linear differential equations as studied by Makarov. Our results sharpen and generalise earlier results of Mahler, Shidlovskii, and Väänänen.
\end{abstract}

\section{Introduction}

Makarov [3] has found lower bounds for linear forms in the values of a certain class of $E$-functions, but the constants involved in his estimates are not given explicitly. In this note we apply the method of Mahler [1]. Firstly, we give an explicit expression for the constant appearing in the lower bound of [3], thereby obtaining an explicit result (Theorem 1). Secondly, the effective transference theorem for Corollary 1.2 is provided by Theorem 2 of the present paper. Corollaries 1.3 and 2.1 of the present paper give explicit results which sharpen those of [10] and Väänänen [9]. We also give some results which sharpen those of [3], [1], Shidlovski $i$ [7] and Väänänen [8] by applying the results of this note to some special $E$-functions. We detail these applications in the last part of the section "Main results".

Received 5 August 1981. The authors are grateful to Professor A.J. van der Poorten for his kind direction and help in connection with the present work. 


\section{Main results}

Let $\mathbb{C}$ be the field of complex numbers, $\mathbb{Z}$ the domain of rational integers, $\mathbb{Q}$ the field of rational numbers, $\mathbb{K}$ an algebraic number field (thus of finite degree over $Q$ ), $0_{\mathbb{K}}$ the domain of integers of $\mathbb{K}$. An entire function $f(z)$ satisfying the following conditions is called an E-function:

(i) $f(z)=\sum_{l=0}^{\infty} \frac{a_{l}}{2 !} z^{2}, a_{l} \in \mathbb{K}, \mid a_{l} \leq c^{2}, z=0,1,2, \ldots$, where $\sqrt{a_{\eta}}$ denotes the maximum of the absolute values of $a_{\imath}$ and its field conjugates, and $c \geqslant 1$ is a positive constant;

(ii) there is a sequence of national numbers $q_{0}, q_{1}, \ldots, q_{l}, \ldots$ such that

$$
q_{2} a_{j} \in 0_{\mathbb{K}}, j=0,1, \ldots, Z, Z=0,1, \ldots,
$$

and

$$
q_{z} \leq c^{2}, \quad z=0,1, \ldots .
$$

The E-functions we consider below are the class of E-functions defined over the field $\mathbb{K}=Q$. Let

$$
f_{i j}(z)=\sum_{i=0}^{\infty} \frac{a_{i j l}}{\imath !} z^{\imath}, i=1, \ldots, k, j=1, \ldots, n_{i},
$$

be a set of E-functions satisfying conditions (i) and (ii) and the following system of the differential equations

(1) $y_{i j}^{\prime}=Q_{i j 0}(z)+\sum_{i=1}^{n_{i}} Q_{i j l}(z) y_{i l}, i=1, \ldots, k, j=1, \ldots, n_{i}$, where $Q_{i j l}(z) \in \mathbb{C}(z), i=1, \ldots, k, j=1, \ldots, n_{i}$. We may, without loss of generality, assume that $Q_{i j l}(z) \in Q(z)$ as is pointed out by Shidlovski i [7].

First of all we introduce some notation. Let $T(z) \in \mathbb{Z}[z]$ be the 
least common denominator of the rational functions $Q_{i j l}(z)$; then

$$
T(z) Q_{i j l}(z) \in \mathbb{Z}[z] \cdot \operatorname{Let}_{g} \max _{\substack{1 \leq i \leq k \\ 1 \leq j \leq n_{i} \\ 0 \leq l \leq n_{i}}}\left(\operatorname{deg} T(z), \operatorname{deg}\left(T(z) Q_{i j l}(z)\right)\right) .
$$

$T$ denotes the maximum of the absolute values of the coefficients of $T(z)$ and the $T(z) Q_{i j l}(z)$. Set $\alpha=a / b \in Q$ such that $\alpha T(\alpha) \neq 0$, where $b>0$. Let

$$
H=\max (|a|, b), B=4 C^{2} H T, L=n_{1}+\ldots+n_{k} .
$$

Denote the minimum value of the orders of the zero at $z=0$ of all the functions $f_{i j}(z)$ by $p$, and their maximum by $q$. We define the constants $\sigma$ and $\sigma_{1}$ as follows:

$\sigma=q, \sigma_{1}=p$ if the set $\left\{f_{i j}(z)\right\}$ and 1 constitute an irreducible set of functions (see [7], p. 389 for the definition), $\sigma=q+\delta, \sigma_{1}=\delta$ otherwise,

where $\delta$ is a constant depending only on the functions $\left\{f_{i j}(z)\right\}$ and the system of differential equations (1). Finally, we define two functions

$$
\begin{aligned}
& c(r)=(L+1)^{2}(g+\sigma+1)(\log B)^{\frac{1}{2}} r(\log r)^{\frac{3}{2}}, \\
& g(r)=e^{-2 c(r)} r ! .
\end{aligned}
$$

We obtain the following results.

THEOREM 1. Let $\left\{f_{i j}(z)\right\}$ be a set of E-functions defined as above which with 1 are linearly independent over $\mathbf{c}(z)$ and satisfy the system of differential equations (1), and let $\left\{x_{i j}\right\} \quad(i=1, \ldots, k$, $\left.j=1, \ldots, n_{i}\right)$ be an arbitrary given set of integers not all zero. Put

$$
x_{i}^{\prime}=\max _{1 \leq j \leq n_{i}}\left(\left|x_{i j}\right|\right), \bar{x}_{i}=\max \left(1, x_{i}^{\prime}\right), x=\max _{1 \leq i \leq k}\left(\bar{x}_{i}\right) \text {. }
$$

If $r$ is the positive integer satisfying the inequality

$$
g(r-1) \leq x<g(r)
$$


then we have

$$
r \geq B^{4(L+1)^{4}(g+\sigma+1)^{2}}+1
$$

and

$$
\prod_{i=1}^{k} \bar{x}_{i}^{n_{i}}\left\|\sum_{i=1}^{k} \sum_{j=1}^{n_{i}} x_{i j} f_{i j}(\alpha)\right\|>e^{-2(L+1) c(r)}
$$

where $\|y\|$ denotes the distance of the real number $y$ from the nearest integer.

COROLLARY 1.1. Under the assumptions of Theorem 1 we have

$$
\prod_{i=1}^{k} \bar{x}_{i}^{n}\left\|_{i=1}^{k} \sum_{j=1}^{n_{i}} x_{i, j} f_{i j}(\alpha)\right\|>x^{-12(L+1)^{3}(g+\sigma+1)(\log B / \log \log x)^{\frac{1}{2}}}
$$

if

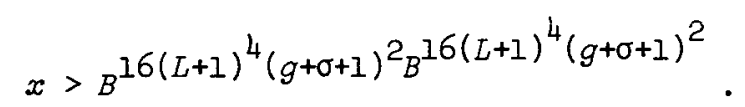

COROLLARY 1.2. Under the hypotheses of Theorem 1 and the conditions $n_{i}=1 \quad(i=1, \ldots, n)$ we have

$$
r \geq B^{4(k+1)^{4}(g+\sigma+1)^{2}}+1
$$

and

$$
\bar{x}_{1} \ldots \bar{x}_{k}\left\|\sum_{i=1}^{k} x_{i} f_{i}(\alpha)\right\|>e^{-2(k+1) c(r)}
$$

COROLLARY 1.3. Under the conditions of Corolzary 1.2 we have

$$
\bar{x}_{1} \ldots \bar{x}_{k}\left\|_{i=1}^{k} x_{i} f_{i}(\alpha)\right\|>x^{-12(k+1)^{3}(g+\sigma+1)(\log B / \log \log x)^{\frac{1}{2}}}
$$

$i f$

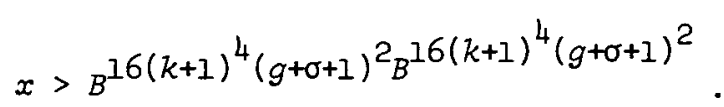

THEOREM 2. Suppose that the functions $f_{i}(z) \quad(i=1, \ldots, k)$, with 1 , beiong to an irreducible set of functions and let $y \geq 2$ be any 
integer. Let $r$ be the positive integer such that $g(r-1) \leq y<g(r)$. under the hypotheses of Corollary 1.2 we have

$$
r \geq B^{4(k+1)^{4}(g+q+1)^{2}}+1
$$

and

$$
y\left\|y f_{1}(\alpha)\right\| \ldots\left\|y f_{k}(\alpha)\right\|>e^{-2(k+2) c(r)} .
$$

COROLLARY 2.1. Under the hypotheses of Theorem 2 we have

$$
y\left\|y f_{1}(\alpha)\right\| \ldots\left\|y f_{k}(\alpha)\right\|>y^{-12(k+2)(k+1)^{2}(g+q+1)(\log B / \log \log y)^{\frac{1}{2}}}
$$

if

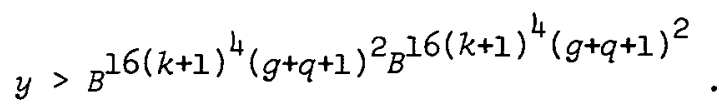

Corollaries 1.3 and 2.1 are similar to Theorems 2 and $2^{\prime}$ in [10] and Theorems 1 and 2 of Väänänen in [9], respectively. The constants here, however, are given in explicit form.

We now mention some examples:

(i) Consider a function

$$
K_{\lambda}(z)=\sum_{h=1}^{\infty} \frac{(-1)^{h}}{h !(\lambda+1) \ldots(\lambda+h)}(z / 2)^{2 h} .
$$

Suppose that $\lambda_{1}, \ldots, \lambda_{m}$ are rational numbers with $\lambda_{i} \neq-1, \pm 1 / 2,-2, \ldots \quad(i=1, \ldots, m)$ and that the $\lambda_{i} \pm \lambda_{2}$

$(I \leq i<l \leq m)$ are not integers. Let $\alpha_{1}, \ldots, \alpha_{n}$ be nonzero rational integers whose squares are distinct. Then the $2 m n$ functions $K_{\lambda_{i}}\left(\alpha_{j} z\right)$, $K_{\lambda_{i}}^{\prime}\left(\alpha_{j} z\right) \quad(1 \leq i \leq m, I \leq j \leq n)$ are a set of $E$-functions which are linearly independent together with the identity over $Q$ (see [3], p. 8) and satisfy the following system of differential equations:

$$
\begin{aligned}
& \frac{d}{d z} y_{1 i j}=y_{2 i j}, \\
& \frac{d}{d z} y_{2 i j}=-\left(2 \lambda_{i}-1\right) z^{-1} y_{2 i j}-\alpha_{j}^{2} y_{1 i j}, i=1, \ldots, m, j=1, \ldots, n .
\end{aligned}
$$


Put

$$
\begin{aligned}
& \lambda_{i}=\lambda_{i}^{\prime} / d \quad(d>0), \quad \lambda^{\prime}=\max _{1 \leq i \leq m}\left(\left|\lambda_{i}^{\prime}\right|\right), \\
& \alpha_{j}=\alpha_{j}^{\prime} / \beta \quad(\beta>0), \quad \alpha=\max _{1 \leq j \leq n}\left(\left|\alpha_{j}^{\prime}\right|\right) .
\end{aligned}
$$

It is easy to compute that

$$
\begin{aligned}
& C=2 \alpha \beta d^{m} e^{6\left(\lambda^{\prime}+d\right) m}, \\
& T=2 \alpha^{2} \beta^{2} d \lambda^{\prime}, \\
& H=1, g=1, L+1=2 m n+1, \\
& \dot{B}=4 C^{2} T H .
\end{aligned}
$$

From Corollary 1.1 we obtain

$\prod_{i=1}^{m} \prod_{j=1}^{n} x_{i j}^{2}\left\|\sum_{i=1}^{m} \sum_{j=1}^{n} x_{i j}{ }^{K} \lambda_{i}\left(\alpha_{j}\right)+y_{i j}{ }^{\prime} \lambda_{i}\left(\alpha_{j}\right)\right\|$

$>X^{-12(2 m n+1)^{3}(2+\sigma)(\log B / \log \log x)^{\frac{1}{2}}}$,

if

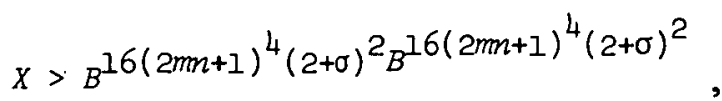

where $x_{i j}, y_{i j}(1 \leq i \leq m, 1 \leq j \leq n)$ are any set of integers not all zero, $\quad X_{i j}=\max \left(I,\left|x_{i j}\right|,\left|y_{i j}\right|\right), \quad x=\max _{1 \leq i \leq m, j \leq j \leq n}\left(x_{i j}\right)$. This result is an application of the theorem of [3], our result however explicitly provides the unspecified constants appearing in [3].

(ii) Suppose that $a_{1}, \ldots, a_{k}$ are distinct rational integers, and that $b>0$ is a rational integer such that $\left(b, a_{1}, \ldots, a_{k}\right)=1$. Consider a set of E-functions

$$
1, e^{\left(a_{1} / b\right) z}, \ldots, e^{\left(a_{k} / b\right) z}
$$

Obviously, the hypotheses of Corollary 1.2 and Theorem 2 are satisfied, and these E-functions belong to an irreducible set of functions, so that $g=0$ and $q=0$. We obtain from Corollary 1.2 and Theorem 2 that 


$$
\begin{aligned}
& \bar{x}_{1} \ldots \bar{x}_{k}\left\|\sum_{i=1}^{k} x_{i} e^{a_{i} / b}\right\|>e^{-2(k+1) c(r)}, \\
& y\left\|y e^{a_{1} / b}\right\| \ldots\left\|y e^{a_{k} / b}\right\|>e^{-2(k+2) c(r)},
\end{aligned}
$$

respectively, where

$$
\begin{aligned}
c(r) & =(k+1)^{2}(\log B)^{\frac{1}{2}} r(\log r)^{\frac{1}{2}}, \\
B & =4 b \max _{1 \leq i \leq k}\left(b,\left|a_{i}\right|\right) .
\end{aligned}
$$

The two inequalities above are similar to those of Theorems 1 and 2 of [1], respectively.+ The exponent $-2(k+2) c(r)$ above constitutes a slight sharpening of the result obtained by Mahler in [1], Theorem 2, which has the exponent $-2 k(k+1) c(r)$.

It follows from Theorem $I^{\prime}$ of [7] that

$$
\begin{aligned}
& \min _{\left|x_{i}\right| \leq x}\left|x_{1} e^{a_{1} / b}+\ldots+x_{k} e^{a_{k} / b}\right|>x^{1-k-\gamma k^{\frac{7}{2}}(\log \log x)^{\frac{1}{2}}} \\
& x_{1}^{2}+\ldots+x_{k}^{2}>0 \\
& x_{i} \in \mathbb{Z}
\end{aligned}
$$

if

$$
x>\exp \left(\exp r^{2} k^{5}\right)
$$

where $\gamma$ is a constant independent of $k$. Our Corollary 1.3 implies: if

$$
x \geq B^{16 k^{4} B^{16 k^{4}}},
$$

then

$$
\begin{aligned}
& \min _{\left|x_{i}\right| \leq x}\left|x_{1} e^{a_{1} / b}+\ldots+x_{k} e^{a_{k} / b}\right|>x^{1-k-12 k^{3}(\log B / \log \log x)^{\frac{1}{2}}}, \\
& x_{1}^{2}+\ldots+x_{k}^{2}>0 \\
& x_{i} \in \mathbb{Z}
\end{aligned}
$$

again sharpening the earlier result.

(iii) Let $\lambda$ be a rational number (but not a rational integer). 
Consider a set of E-functions

$$
\phi_{\lambda}\left(\left(a_{1} / b\right) z\right), \ldots, \phi_{\lambda}\left(\left(a_{k} / b\right) z\right),
$$

where

$$
\phi_{\lambda}(z)=\sum_{l=0}^{\infty} \frac{z^{2}}{(\lambda+1) \ldots(\lambda+l)}
$$

By [5], $\phi_{\lambda}\left(\left(a_{1} / b\right) z\right), \ldots, \phi_{\lambda}\left(\left(a_{k} / b\right) z\right)$, with 1 , belong to an irreducible set of functions which are linearly independent over $\mathbb{C}(z)$ and satisfy the following system of differential equations:

$$
\frac{d}{d z} \phi_{\lambda}\left(\left(a_{i} / b\right) z\right)=\lambda / z+\left(\left(a_{i} / b\right)-(\lambda / z)\right) \phi_{\lambda}\left(\left(a_{i} / b\right) z\right), \quad i=1, \ldots, k .
$$

Put $\lambda=\lambda_{1} / d, \quad\left(\lambda_{1}, d\right)=1, d>0, \alpha=1$. It is easy to compute that

$$
\begin{aligned}
& C=b d^{2} e^{12\left(\left|\lambda_{1}\right|+d\right)} \max _{1 \leq i \leq k}\left(\left|a_{i}\right|\right) \text { (by Mahler [2], p. 146), } \\
& T=b d \lambda_{1} \max _{1 \leq i \leq k}\left(\left|a_{i}\right|\right), \\
& H=1, g=1, q=0 .
\end{aligned}
$$

From Corollaries 1.3 and 2.1 we obtain

$$
\bar{x}_{1} \ldots \bar{x}_{k}\left\|\sum_{i=1}^{k} x_{i} \phi_{\lambda}\left(a_{i} / b\right)\right\|>x^{-24(k+1)^{3}(\log B / \log \log x)^{\frac{1}{2}}}
$$

if

$$
x>B^{64(k+1)^{4} B^{64(k+1)^{4}}},
$$

and

$$
y\left\|y \phi_{\lambda}\left(a_{1} / b\right)\right\| \ldots\left\|y \phi_{\lambda}\left(a_{k} / b\right)\right\|>y^{-24(k+1)^{2}(k+2)(\log B / \log \log y)^{\frac{1}{2}}}
$$

if

$$
y>B^{64(k+1)^{4} B^{64(k+1)^{4}}} .
$$


These results are Theorems $I$ and 2 of [8], respectively; however we explicitly compute the unspecified constants that appear in [8].

\section{Lemmas}

LEMMA 1. Let $\left(g_{i j}\right) \quad(1 \leq i \leq m, 1 \leq j \leq n)$ be a $m \times n \quad(m<n)$ matrix of integers. Put

$$
G_{i}=\sum_{j=1}^{n}\left|g_{i j}\right|, \quad i=1, \ldots, m .
$$

Then there are integers $x_{1}, \ldots, x_{n}$ not all zero such that

$$
\sum_{j=1}^{n} g_{i j} x_{j}=0, \quad i=1, \ldots, m,
$$

and

$$
\max _{1 \leq i \leq n}\left(\left|x_{i}\right|\right) \leq\left(G_{1} \ldots G_{m}\right)^{1 /(n-m)} \text {. }
$$

This is Lemma 1 of [1].

LEMMA 2. Let $r_{1}, \ldots, r_{k}$ and $R$ be positive integers satisfying

$$
\begin{gathered}
r=r_{0}=\max \left(r_{1}, \ldots, r_{k}\right) \geq 2, \\
\left.L<R \leq \sum_{i=0}^{k} n_{i} r_{i}+L \quad \text { (where } n_{0}=1\right) ;
\end{gathered}
$$

and let

$$
\begin{aligned}
& m=\sum_{i=0}^{k} n_{i} r_{i}+L+1-R, n=\sum_{i=0}^{k} n_{i} r_{i}+L+1, \\
& M=\left[(L+1)^{m}\left(2 c^{2}\right)^{m(m-1) / 2}\right]^{1 / R} .
\end{aligned}
$$

Then there are polynomials $P_{i j}(z) \in \mathbb{Z}[z] \quad(i=0,1, \ldots, k$, $\left.j=1, \ldots, n_{i}\right\}$ which do not all vanish identically and have the following properties:

(i) $\operatorname{deg} P_{i j}(z) \leq r$, ord $P_{i j}(z) \geq r-r_{i},\left|P_{i j}\right| \leq r_{i} ! 2^{r} M$, 
$i=0,1, \ldots, k, j=1, \ldots, n_{i}$, where ord $P_{i j}(z)$

denotes the order of the zero at $z=0$ of the polynomial $P_{i j}(z)$, and $\mid P_{i j}$ denotes the height of $P_{i j}(z)$, that is, the maximum of the absolute values of the coefficients of $P_{i j}(z)$;

(ii) let

$$
\begin{aligned}
F(z) & =\sum_{i=0}^{k} \sum_{j=1}^{n} P_{i j}(z) f_{i j}(z) \quad\left(\text { where } f_{01}(z) \equiv 1\right) \\
& =\sum_{h=m}^{\infty} r ! \sigma_{h}(h !)^{-1} z^{h}=\sum_{h=m}^{\infty} \rho_{h} z^{h},
\end{aligned}
$$

then

$$
\left|\rho_{h}\right| \leq(L+1) r !(h !)^{-1}(1+C)^{h}, \quad h \geq m
$$

Proof. Let $S$ be the set $S=\left\{(i, l) \mid 0 \leq i \leq k, r_{i} r_{i} \leq \leq \leq r\right\}$, and write

$$
P_{i j}(z)=r ! \sum_{i=0}^{r} p_{i j l}(2 !)^{-1} z, i=0,1, \ldots, k, j=1, \ldots, n_{i} .
$$

Property $(i)$ implies $p_{i j l}=0$ if $(i, z) \bar{\epsilon} S$ and $j=1, \ldots, n_{i}$. Property $(i i)$ implies ord $F(z) \geq m$. Thus the $p_{i j z^{\prime}}$ s satisfy the following system of equations

$$
p_{01 h}+\sum_{i=1}^{k} \sum_{j=1}^{n_{i}} \sum_{l=0}^{h}\left(\begin{array}{l}
h \\
l
\end{array}\right) a_{i, j, h-l p_{i j l}}=0, h=0,1, \ldots, m-1 .
$$

On multiplying these $m$ equations by $q_{0}, q_{1}, \ldots, q_{m-1}$, respectively, we obtain

(6) $q_{h} p_{01 h}+\sum_{i=1}^{k} \sum_{j=1}^{n_{i}} \sum_{i=0}^{h}\left(\begin{array}{l}h \\ l\end{array}\right) q_{h} a_{i, j, h-2} p_{i j l}=0, h=0,1, \ldots, m-1$.

This is a system of linear equations in the unknowns $\left\{p_{i j l}\right\}$ and with rational integer coefficients. Put 


$$
G_{h}=q_{h}+\sum_{i=1}^{k} \sum_{j=1}^{n_{i}} \sum_{i=0}^{h}\left(\begin{array}{l}
h \\
l
\end{array}\right)\left|q_{h} a_{i, j, h-l}\right| .
$$

Clearly, we have

$$
G_{h} \leq(L+1)\left(2 c^{2}\right)^{h}, h=0,1, \ldots, m-1
$$

The number of unknowns for the system of equations (6) is equal to $n>m$. So we see from Lemma 1 that this system of equations has a set of rational integer solutions $\left\{p_{i j l}\right\}$ not all zero and satisfying

$$
\begin{aligned}
\max _{i, j, \ell}\left|p_{i j l}\right| & \leq\left(G_{0} \ldots G_{m-1}\right)^{1 /(n-m)} \\
& \leq\left[(L+1)^{m}\left(2 C^{2}\right)^{m(m-1) / 2}\right]^{1 / R}=M .
\end{aligned}
$$

Since

$$
P_{i j}(z)=r_{i} ! \sum_{i=0}^{r} r !\left(r_{i} ! z_{l}\right)^{-1} p_{i j l^{2}} z^{2},
$$

it follows that

$$
\mid P_{i j} \leq r_{i} ! 2^{r} M, \quad i=0,1, \ldots, k, j=1, \ldots, n_{i} .
$$

Because

$$
\sigma_{h}=p_{01 h}+\sum_{i=1}^{k} \sum_{j=1}^{n_{i}} \sum_{l=0}^{h}\left(\begin{array}{l}
h \\
l
\end{array}\right) \alpha_{i, j, h-l} p_{i j l},
$$

we have

$$
\left|\rho_{h}\right|=(r ! / h !)\left|\sigma_{h}\right| \leq(L+1) r !(h !)^{-1}(1+C)^{h} M
$$

completing the proof of the lemma.

Let

$$
F_{0}(z)=F(z), \quad F_{\tau}(z)=T(z) \frac{d}{d z} E_{\tau-1}(z), \quad \tau=1,2, \ldots .
$$

It follows from the system of differential equations (1) and (7) that 


$$
F_{\tau}(z)=\sum_{i=0}^{k} \sum_{j=1}^{n_{i}} P_{i j \tau}(z) f_{i j}(z)
$$

where $P_{i j \tau}(z)$ satisfy the following recurrence relations:

$$
\begin{aligned}
& P_{i j 0}(z)=P_{i j}(z), \\
& P_{01 \tau}(z)=T(z) \frac{d}{d z} P_{0,1, \tau-1}(z)+\sum_{i=1}^{k} \sum_{j=1}^{n_{i}} T(z) Q_{i j 0}(z) P_{i, j, \tau-1}(z), \\
& P_{i j \tau}(z)=T(z) \frac{d}{d z} P_{i, j, \tau-1}(z)+\sum_{i=1}^{n_{i}} T(z) Q_{i \tau_{j}}(z) P_{i, \tau, \tau-1}(z), \\
& i=1, \ldots, k, j=1, \ldots, n_{i} .
\end{aligned}
$$

Clearly, $P_{i j \tau}(z) \in \mathbb{Z}[z]$. Further, put

$$
\begin{array}{cc}
\tilde{f}_{0}(z)=f_{0 I}(z) \equiv I, & \tilde{f}_{V}(z)=f_{i j}(z), \\
\tilde{P}_{\tau 0}(z)=P_{0 I \tau}(z), & \tilde{P}_{\tau \nu}(z)=P_{i j \tau}(z),
\end{array}
$$

where

$$
v=v(i, j)=\sum_{i=0}^{i-1} n_{l}+j-1, i=1, \ldots, k, j=1, \ldots, n_{i} .
$$

In particular, set $v=0$ if $i=0$ (of course $j=1$ ). Conversely, if $v$ is given, then we can determine $i$ by the following inequality

$$
\sum_{i=0}^{i-1} n_{i} \leq v \leq \sum_{i=0}^{i} n_{i}-1 \text {. }
$$

Let $P(z)$ be the matrix

$$
P(z)=\left(\tilde{P}_{\tau \nu}(z)\right)_{0 \leq \tau, \nu \leq L},
$$

and put

$$
\Delta(z)=\operatorname{det} P(z) .
$$

LEMMA 3. Let $\left\{f_{i j}(z)\right\}$ be a set of E-functions defined as above which satisfy the system of differential equations (1) and which, with 1 , are linear independent over $\mathbb{C}(z)$. Then there exists a constant 


$$
N_{0}=L(L+1)(g+1) / 2+R+\sigma_{1}
$$

such that when $r^{*}=\min \left(r_{1}, \ldots, r_{k}\right)>N_{0}$ we have $\Delta(z) \neq 0$, and

$$
\Delta(z)=z^{(L+1) r-R-(L(L+1) / 2)-p_{\Delta_{1}}(z),}
$$

where $\Delta_{1}(z) \neq 0, \Delta_{1}(z) \in \mathbb{Z}[z]$ and

$$
\operatorname{deg} \Delta_{1}(z) \leq t=R+L(L+1)(g+1) / 2+p
$$

Proof. Suppose that the rank of $\Delta(z)$ is $W+1<L+1$. Obviously $W \geq 0$. Then there is at least one non-zero minor determinant of order $W+1$ in $P(z)$. Without loss of generality, we assume that it is in the left upper corner, namely that it is the principal minor determinant $\Delta_{0}(z) \neq 0$. Then there exists a set of rational functions

$$
D_{\omega \nu}(z) \in Q(z), \omega=0,1, \ldots, W, \nu=W+1, \ldots, L,
$$

such that

$$
\tilde{P}_{\tau \nu}(z)=\sum_{\omega=0}^{W} \tilde{P}_{\tau \omega}(z) D_{\omega \nu}(z), \tau=0,1, \ldots, W, \nu=W+1, \ldots, L
$$

(see Lemma 6 in [6]). $F_{\tau}(z)$ can be rewritten as

$$
F_{\tau}(z)=\sum_{\nu=0}^{L} \tilde{P}_{\tau \nu}(z) \tilde{f}_{\nu}(z)
$$

and we have

$$
F_{\tau}(z)=\sum_{v=0}^{W} \tilde{P}_{\tau \nu}(z) u_{v}(z), \tau=0,1, \ldots, W
$$

where

$$
u_{v}(z)=\tilde{f}_{v}(z)+\sum_{\omega=W+1}^{L} \tilde{f}_{\omega}(z) D_{v \omega}(z)
$$

Let $T_{1}(z)$ be the least common denominator of the $D_{\omega \nu}(z)$. Put

$$
U_{v}(z)=T_{1}(z) u_{v}(z), v=0,1, \ldots, W \text {. }
$$

From Lemma 6 in [6] and Lemma 4 in [5] we find that 


$$
\operatorname{ord} U_{v}(z) \leq \sigma_{1}
$$

In view of (10), we get

$$
\Delta_{0}(z) U_{v}(z)=\sum_{\omega=0}^{W} T_{1}(z) \Delta_{\omega v}(z) F_{\omega}(z),
$$

where $\Delta_{\omega \nu}(z)$ is the cofactor of the element $\tilde{P}_{\omega \nu}(z)$ of the matrix corresponding to $\Delta_{0}(z)$. It is easy to compute that

$$
\begin{aligned}
\operatorname{ord}\left(\Delta_{0}(z) U_{\nu}(z)\right) & \leq \operatorname{deg} \Delta_{0}(z)+\operatorname{ord} U_{\nu}(z) \\
& \leq(W+1) r+L(L+1) g / 2+\sigma_{1}, \\
\text { ord } \tilde{P}_{\tau \nu}(z)>r-r_{i(\nu)}-\tau, & >\operatorname{ord} F_{0}(z)-L \geq m-L, \\
\text { ord } F_{\tau}(z) & >\sum_{0 \leq \tau \leq W} r_{i(\tau)}-L(L+1) / 2, \\
\text { ord } \Delta_{\omega \nu}(z) & \geq \underset{\tau \neq \nu}{ }
\end{aligned}
$$

where $i=i(\tau)$ satisfies (8). It follows from (11) that

$$
(W+1) r+L(L+1) g / 2+\sigma_{1}>W r-\sum_{\substack{0 \leq \tau \leq W \\ \tau \neq U}} r_{i(\tau)}-L(L+1) / 2+m-L .
$$

Since $W<L$ this inequality implies that there exists at least one suffix $i(\tau)$ in the interval $0 \leq i(\tau) \leq k$ such that

$$
r_{i(\tau)}<L(L+1)(g+1) / 2+R+\sigma_{1} .
$$

This contradicts the assumption of the lemma. Hence we must have $W=L$, that is, $\Delta(z) \neq 0$.

Without loss of generality, we suppose that

$$
\text { ord } f_{\nu_{0}}(z)=\text { ord } f_{i_{0} j_{0}}(z)=p
$$

for some $v_{0}=v\left(i_{0}, j_{0}\right)$. By (9) we obtain

$$
\Delta(z) \tilde{f}_{v_{0}}(z)=\sum_{\omega=0}^{L} F_{\omega}(z) \Delta_{\omega v_{0}}(z)
$$


Thus

$$
\text { ord } \begin{aligned}
\Delta(z) & \geq \min _{\substack{0 \leq \omega \leq L\\
}}\left(\operatorname{ord} F_{\omega}(z)+\operatorname{ord} \Delta_{\omega \nu_{0}}(z)\right)-\operatorname{ord} \tilde{f}_{\nu_{0}}(z) \\
& \geq m-L+r L-\sum_{\substack{0 \leq \tau \leq L \\
\tau \neq \nu_{0}}} r_{i(\tau)}-L(L+1) / 2-p \\
& \geq(L+1) r-R-L(L+1) / 2-p,
\end{aligned}
$$

and therefore

$$
\begin{aligned}
\operatorname{deg} \Delta_{1}(z) & \leq \operatorname{deg} \Delta(z)-\operatorname{ord} \Delta(z) \\
& \leq(L+1) r+L(L+1) g / 2-(L+1) r+R+L(L+1) / 2+p \\
& =R+L(L+1)(g+1) / 2+p=t,
\end{aligned}
$$

completing the proof of the lemma.

LEMMA 4. Under the assumptions of Lemma 3 there exist $L+1$ suffixes $J(\tau) \quad(0 \leq \tau \leq L)$ such that

$$
0 \leq J(0)<J(1)<\ldots<J(L) \leq L+t
$$

and

$$
\operatorname{det}\left(\tilde{P}_{J(\tau), \nu}(\alpha)\right)_{0 \leq \tau, \nu \leq L} \neq 0
$$

The proof of this lemma is similar to Lemma 7 of [6].

LEMMA 5. Under the hypotheses of Lemma 4 there exist $(L+1)^{2}$ rational integers $q_{\tau \nu}(0 \leq \tau, \nu \leq L)$ with the following properties:

(i) $\operatorname{det}\left(q_{\tau \nu}\right)_{0 \leq \tau, v \leq I} \neq 0$;

(ii) for each pair $(\tau, \nu)$ we have

$$
\left|q_{\tau \nu}\right| \leq c_{1} r_{i(\nu)} !
$$

where $i=i(v)$ satisfies (8) and

$$
C_{1}=2^{r}[(L+t) g+r+L]^{L+t} T^{L+t}(2 H)^{r+(L+t)} g_{M} ;
$$

(iii) for $\tau=0,1, \ldots, L$, we have

$$
\left|\sum_{v=0}^{L} q_{\tau \nu} \tilde{f}_{v}(\alpha)\right| \leq c_{2}\left(\prod_{i=1}^{k}\left(r_{i} !\right)^{n_{i}}\right)^{-1},
$$


where

$$
\begin{gathered}
C_{2}=(L+1)[(L+t) g]^{L+t}(2 H)^{2(L+t) g_{H}(L+2) r}(2 T)^{L+t}[(L+1) r]^{2 t}(1+C){ }^{(L+1) r} e^{2 C H} M . \\
\text { Proof. Clearly } \\
\qquad \operatorname{deg} \tilde{P}_{J(\tau), v}(z) \leq r+J(\tau) g \leq r+(L+t) g .
\end{gathered}
$$

Put

$$
q_{\tau \nu}=b^{r+(L+t)} g_{\tilde{P}_{J(\tau), \nu}}(\alpha)
$$

Thus all the $q_{\tau \nu}$ are rational integers. It follows from Lemma 4 that

$$
\operatorname{det}\left(q_{\tau \nu}\right)_{0 \leq \tau, \nu \leq L} \neq 0
$$

Now consider two power series

$$
U(z)=\sum_{i=0}^{\infty} u_{i} z^{l} \text { and } V(z)=\sum_{i=0}^{\infty} v_{i} z^{z}
$$

$V(z)$ is said to majorize the series $U(z)$ if

$$
v_{\imath} \geq 0, \quad\left|u_{\eta}\right| \leq v_{\eta}, \quad \imath=0,1, \ldots .
$$

We write $U(z) \ll V(z)$.

It is not difficult to verify by induction that

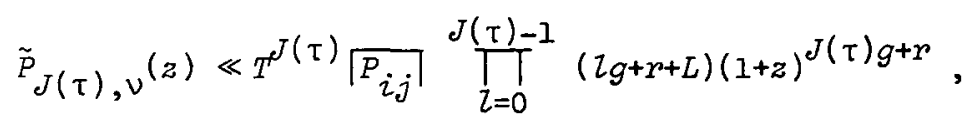

where $(i, j)$ corresponds to the suffix $\nu$. Thus

$$
\begin{aligned}
\left|q_{\tau \nu}\right| & \leq\left. b^{r+(L+t) g_{T} J(\tau)}\right|_{i j}\left[[(J(\tau)-1) g+r+L]^{J(\tau)}(1+|a| / b)^{J(\tau) g+r}\right. \\
& \leq T^{L+t}{ }_{P_{i j}}[(L+t) g+r+L]^{L+t}(2 H) \\
& \leq C_{1} r_{i(\nu)} ! .
\end{aligned}
$$

In view of (9), we see, again by induction, that 


$$
\begin{aligned}
F_{J(\tau)}(z) & \ll T^{J(\tau)}(1+z)^{J(\tau) g} \prod_{l=0}^{J(\tau)-1}(2 g+(d / d z)) F_{0}(z) \\
& \ll T^{J(\tau)}(J(\tau) g)^{J(\tau)}(1+z)^{J(\tau) g}(1+(d / d z))^{J(\tau)} F_{0}(z) \\
& \ll(2 T)^{J(\tau)}(J(\tau) g)^{J(\tau)}(1+z)^{J(\tau) g} \sum_{h=m}^{\infty} \frac{r !\left|\sigma_{h}\right|}{(h-J(\tau)) !} z^{h-J(\tau)} .
\end{aligned}
$$

\section{Hence}

$$
\begin{aligned}
& \left|\sum_{\nu=0}^{L} q_{\tau \nu} \tilde{f}_{\nu}(\alpha)\right| \\
& \leq b^{r+(L+t) g}\left|F_{J(\tau)}(a / b)\right| \\
& \leq r !(2 T)^{J(\tau)}(J(\tau) g)^{J(\tau)} b^{r+(L+t) g}(1+|a| / b)^{J(\tau) g} \sum_{h=m}^{\infty} \frac{\left|\sigma_{h}\right|}{(h-J(\tau)) !}(|a| / b)^{h-J(\tau)} \\
& \leq(L+1) r !(2 T)^{L+t}[(L+t) g]^{L+t}(1+C)^{m_{b} r+(L+t) g}(1+|a| / b) \\
& \quad \cdot(\mid a+t) g \\
& \leq(L+1) r ![(L+t) g]^{L+t}(2 H)^{2(L+t) g_{H}(L+2) r}(2 T)^{L+t} \cdot e^{2 C H}(1+C)^{(L+1) r} M \cdot[(m-J(\tau)) !]^{-1} .
\end{aligned}
$$

On the other hand, we have

$$
\begin{aligned}
(m-J(\tau)) ! & \geq[m-(L+t)] ! \geq\left(\sum_{i=0}^{k} n_{i} r_{i}-2 t\right) ! \\
& \geq\left(\sum_{i=0}^{k} n_{i} r_{i}\right) ![(L+1) r]^{-2 t} .
\end{aligned}
$$

Therefore

$$
\begin{aligned}
& \left|\sum_{\nu=0}^{L} q_{\tau \nu} \tilde{f}_{\nu}(\alpha)\right| \leq(L+1)[(L+t) g]^{L+t}(2 H)^{2(L+t) g_{H}(L+2) r}(2 T)^{L+t} \\
& \text { - }[(L+1) r]^{2 t}(1+C)^{(L+1) r} e^{2 C H} M\left(\prod_{i=1}^{k}\left(r_{i} !\right)^{n} i\right)^{-1} \\
& \leq c_{2}\left(\prod_{i=1}^{k}\left(r_{i} !\right)^{n}\right)^{-1}
\end{aligned}
$$

completing the proof. 
LEMMA 6. Let

$$
L_{i}(X)=\sum_{j=1}^{k} \alpha_{i j} x_{j}, \quad i=1, \ldots, k,
$$

be $k$ linearly independent linear forms, and let

$$
M_{i}(y)=\sum_{j=1}^{k} B_{i j} y_{j}, \quad i=1, \ldots, k,
$$

be a further $k$ inear forms. Suppose that

$$
\sum_{i=1}^{k} L_{i}(X) M_{i}(Y)=X Y
$$

holds identically for $X, Y \in \mathbf{R}^{k}$. Let $\lambda_{1}, \ldots, \lambda_{k}$ denote the successive minima of the parelzelepiped defined by $\left|L_{i}(X)\right| \leq 1$ ( $\left.1 \leq i \leq k\right)$. Denote by $v_{1}, \ldots, v_{k}$ the successive minima of the parallelepiped defined by $\left|M_{i}(Y)\right| \leq 1 \quad(1 \leq i \leq k)$. Then we have

$$
\lambda_{i} \nu_{k+1-i} \geq 1 / k, \quad i=1, \ldots, k
$$

See Lemma 1 of [10] for the proof of this lemma.

\section{Proof of Theorem 1}

Let $r$ be a positive integer satisfying (2). From Stirling's formula

$$
r !=(2 \pi r)^{\frac{1}{2}} r^{r} e^{-r+\rho(r)}, 0<\rho(r)<1 /(12 r),
$$

we obtain that

$$
\log g(r) / r=\log r-2(L+1)^{2}(g+\sigma+1)(\log B)^{\frac{1}{2}}(\log r)^{\frac{1}{2}}-1+\sigma(r),
$$

where

$$
\sigma(r)=\frac{\log r}{2 r}+\frac{\log 2 \pi}{2 r}+\frac{\rho(r)}{r}
$$

It is easily verified that $0<\sigma(r)<1$ for $r \geq 2$. Hence $\log r-2(L+1)^{2}(g+\sigma+1)(\log B)^{\frac{1}{2}}(\log r)^{\frac{1}{2}}-1<\log g(r) / r$

$$
<\log r-2(L+1)^{2}(g+\sigma+1)(\log B)^{\frac{1}{2}}(\log r)^{\frac{1}{2}} .
$$


From the definition of $g(r)$ and this inequality, we can immediately verify that

$$
g(1)=1 ; g(r)<1 \text { if } 2 \leq \dot{r} \leq B^{4(L+1)^{4}(g+\sigma+1)^{2}} .
$$

Because $c(r)$ is a strictly increasing function of $r$ (when $r \geq 2$ ) and $g(r)>x \geq 1$, it follows that $r$ must satisfy

$$
r \geq B^{4(L+1)^{4}(g+\sigma+1)^{2}}+1
$$

thus the inequality (3) holds. By the definition of $r$, we also have

$$
(r-1) ! \leq e^{2 c(r)} x<r ! \text {. }
$$

Similarly, define the integers $r_{1}, \ldots, r_{k}$ by the inequalities

$$
\left(r_{i}-1\right) ! \leq e^{2 c(r)} \bar{x}_{i} \leq r_{i} !, i=1, \ldots, k \text {. }
$$

Clearly, the inequalities (14), (15) imply $r=\max \left(r_{1}, \ldots, r_{k}\right) \cdot$ Now write

$$
R=\left[(L+1) r(\log B / \log r)^{\frac{1}{2}}\right]+1 \text {, }
$$

where $[y]$ denotes the integer part of $y$. Because of the inequality (3), and noting that $r /(\log r)^{\frac{1}{2}}$ is an increasing function of $r$, we easily verify that

$$
L<R \leq \sum_{i=0}^{k} n_{i} r_{i}+L .
$$

We now show that $r^{*}=\min \left(r_{1}, \ldots, r_{k}\right)>N_{0}$. We have

$$
\begin{gathered}
\log r>4(L+1)^{4}(g+\sigma+1)^{2} \log B, \\
R>(L+1) r(\log B / \log r)^{\frac{1}{2}},
\end{gathered}
$$

by (3) and (16). So $2 R>N_{0}$. If we were to assume that some $r_{i} \leq N_{0}$, then we have the inequality

$$
\log \left(r_{i} !\right)<r_{i} \log r_{i}<2 R \log 2 R \leq 2 c(r)
$$

and so $r_{i} !<e^{2 c(r)}$. This is contrary to the definition of $r_{i}$, hence 
certainly $r^{*}>N_{0}$. Thus we have verified that $r, r_{1}, \ldots, r_{k}$ and $R$ satisfy the conditions of Lemmas 2 and 3 .

By Lemma 5, we have obtained $(L+1)^{2}$ integers $q_{\tau \nu}(0 \leq \tau, \nu \leq L)$ satisfying $\operatorname{det}\left(q_{\tau v}\right) \neq 0$. Further let $\left\{x_{i j}\right\} \quad\left(1 \leq i \leq k, 1 \leq j \leq n_{i}\right)$ be a set of integers satisfying the hypotheses of Theorem 1 , and let $b$ be any integer. Then we can form a $(L+1) \times(L+1)$ determinant which does not vanish; without loss of generality, we may assume that, say,

$$
D=\left|\begin{array}{cccc}
b & x_{11} & \ldots & x_{k n_{k}} \\
q_{10} & q_{11} & \ldots & q_{1 L} \\
\ldots \ldots & \ldots & \ldots & \ldots \\
q_{L 0} & q_{L 1} & \ldots & q_{L L}
\end{array}\right| \neq 0 .
$$

Let

$$
\begin{aligned}
& L_{0}=b+\sum_{i=1}^{k} \sum_{j=1}^{n_{i}} x_{i j} f_{i j}(\alpha), \\
& L_{\tau}=\sum_{\nu=0}^{L} q_{\tau \nu} \tilde{f}_{\nu}(\alpha), \tau=1, \ldots, L .
\end{aligned}
$$

Thus $D$ can be rewritten as

$$
D=\left|\begin{array}{cccc}
L_{0} & x_{11} & \ldots & x_{k n_{k}} \\
L_{1} & q_{11} & \ldots & q_{1 L} \\
\ldots & \ldots & \ldots & \ldots \\
L_{L} & q_{L 1} & \ldots & q_{L L}
\end{array}\right|
$$

Decomposing this determinant according to the first column, we obtain

$$
D=L_{0} M_{0}+L_{1} M_{1}+\ldots+L_{L} M_{L}
$$

where $M_{i}$ is the cofactor of $L_{i}, i=0,1, \ldots, L$. By (12) and (13) of Lemma 5, we have 


$$
\begin{aligned}
\left|M_{0}\right| & \leq L ! c_{1}^{L} \prod_{i=1}^{k}\left(r_{i} !\right)^{n_{i}}, \\
\left|M_{\tau}\right| & \leq(L-1) ! c_{1}^{L-1} \prod_{i=1}^{k}\left(r_{i} !\right)^{n_{i}} \sum_{i=1}^{k} \sum_{j=1}^{n_{i}} \frac{\left|x_{i j}\right|}{r_{i} !} \\
& \leq(L-1) ! c_{1}^{L-1} \prod_{i=1}^{k}\left(r_{i} !\right)^{n_{i}} \sum_{i=1}^{k} \frac{n_{i} \bar{x}_{i}}{r_{i} !} \\
\left|L_{\tau}\right| & \leq c_{2}\left(\prod_{i=1}^{k}\left(r_{i} !\right)^{n_{i}}\right)^{-1}, \tau=1, \ldots, L .
\end{aligned}
$$

But $D$ is a non-zero integer, so $|D| \geq 1$. Therefore we have

$$
\begin{aligned}
I \leq|D| & \leq L ! C_{1}^{L} \prod_{i=1}^{k}\left(r_{i} !\right)^{n}{ }^{n}\left|L_{0}\right|+L ! C_{1}^{L-1} C_{2} \sum_{i=1}^{k} \frac{n_{i} \bar{x}_{i}}{r_{i}} \\
& =U+V .
\end{aligned}
$$

According to the definition of $r_{i}$, we have

$$
\begin{aligned}
& \prod_{i=1}^{k}\left(r_{i} !\right)^{n} \leq r^{L} e^{2 L c(r)} \prod_{i=1}^{k} \bar{x}_{i}^{n}, \\
& \sum_{i=1}^{k} \frac{n_{i} \bar{x}_{i}}{r_{i} !} \leq L e^{-2 c(r)} .
\end{aligned}
$$

Thus

$$
\begin{aligned}
& 2 U \leq 2 L ! C_{1}^{L} r^{L} e^{2 L c(r)}\left|L_{0}\right| \prod_{i=1}^{k} \bar{x}_{i}^{n}, \\
& 2 V \leq 2(L+1) ! C_{I}^{L-1} C_{2} e^{-2 c(r)} .
\end{aligned}
$$

We shall next establish upper estimates for (18) and (19). By using (3) and the definition of $R$, we can easily obtain the following inequalities:

$$
\begin{aligned}
L+t & =L+R+L(L+1)(g+1) / 2+p \leq R+L(L+1)(g+1)+\sigma, \\
(L+t) g+r+L=g[R+L(L+1)(g+1)+\sigma]+r+L & \leq(g+2) r, \\
(L+t) g & \leq(g+1) r .
\end{aligned}
$$




\section{Moreover}

$$
\begin{aligned}
C_{1} \leq 2^{r} T^{R+L(L+1)(g+1)+\sigma}[(g+2) r]^{R+L(L+1)(g+1)+\sigma} & \\
& \cdot(2 H)^{r+g[R+L(L+1)(g+1)+\sigma]}(L+1){ }^{(L+1) r / R}\left(2 C^{2}\right)^{(L+1)^{2} r^{2} /(2 R) .}
\end{aligned}
$$

It follows that

$$
\begin{array}{r}
2 L ! C_{1}^{L} r^{L} \leq\left(2 C^{2}\right)^{L(L+1)^{2} r^{2} /(2 R)}{ }_{r}^{L(R-1)} \cdot L^{L} \cdot(2 T)^{L r} \cdot{ }^{L^{2}(L+1)(g+1)+L \sigma} \\
\cdot(2 H)^{L r+L g[R+L(L+1)(g+1)+\sigma]} \cdot(g+2)^{L[R+L(L+1)(g+1)+\sigma]} \\
\cdot r^{L^{2}(L+1)(g+1)+L(\sigma+2)} \cdot(L+1)^{L(L+1) r / R} .
\end{array}
$$

Clearly, we have, by (16),

$$
\left(2 C^{2}\right)^{L(L+1)^{2} r^{2} /(2 R)} x^{L(R-1)} \leq e^{(3 / 2) c(x)} .
$$

Since $B \geq 4, L \geq 1, g \geq 0, \sigma \geq 0$, we have $r \geq B^{64} \geq 2^{128}$ by (3). It follows that

$$
(\log r) / r \leq 128(\log 2) / 2^{128}<2^{-120} \text {, }
$$

since $(\log r) / r$ is a strictly decreasing function of $r$ (when $r \geq 2$ ). Thus we can obtain the following inequalities by simple calculation:

$$
\begin{aligned}
\frac{L \log L}{c(r)} & \leq \frac{L^{2}}{2(L+1)^{4} r \log B}<2^{-7} ; \\
\frac{L r \log 2 T}{c(r)} & \leq \frac{L r \log B}{2(L+1)^{4} r \log B}<2^{-4} ; \\
\frac{\left[L^{2}(L+1)(g+1)+L \sigma\right] \log T}{c(r)} & \leq \frac{L(L+1)^{2}(g+1+\sigma) \log B}{2(L+1)^{4}(g+\sigma+1)^{2} r \log B}<2^{-7} ;
\end{aligned}
$$

$\frac{\{L r+L g[R+L(L+1)(g+1)+\sigma]\}] \log 2 H}{c(r)}$

$$
\begin{aligned}
& \leq \frac{L r \log B}{2(L+1)^{4} r \log B}+\frac{L g(L+1) \log B}{(L+1)^{2}(g+\sigma+1) \log x}+\frac{L g \log B}{(L+1)^{2}(\log B)^{\frac{1}{2}} r(\log r)^{\frac{1}{2}}} \\
& +\frac{\left[L^{2} g(L+1)^{2}(g+1)+\operatorname{Lg} \sigma\right] \log B}{2(L+1)^{4}(g+\sigma+1)^{2} r \log B} \\
& \leq 2^{-4}+2^{-6}+2^{-132}+2^{-130} \leq 2^{-3} ;
\end{aligned}
$$




$$
\begin{aligned}
& \frac{L[R+L(L+1)(g+1)+\sigma] \log (g+2)}{c(r)} \leq \frac{L R \log (g+2)}{c(r)}+\frac{L(L+1)^{2}(g+\sigma+1) \log (g+2)}{c(r)} \\
& \leq \frac{L R(g+1)}{c(r)}+\frac{L(L+1)^{2}(g+\sigma+1)^{2}}{c(r)} \\
& \leq 1 /(\log r)+1 /\left(2(L+1)^{3} r\right)+1 /(2(L+1) r) \\
& \leq 2^{-7}+2^{-132}+2^{-130} \leq 2^{-6} \text {; } \\
& \frac{L\left[(L+1)^{2}(g+1)+\sigma+2\right] \log r}{c(r)} \leq \frac{L(L+1)^{2}(g+\sigma+1) \log r}{c(r)}+\frac{2 L \log r}{c(r)} \\
& \leq \frac{L}{2(L+1)^{2} \log B} \quad \frac{\log r}{r}+\frac{2 L}{2(L+1)^{4} \log B} \quad \frac{\log r}{r} \\
& \leq 2^{-132}+2^{-123} \leq 2^{-7} ; \\
& \frac{L(L+1) r \log (L+1)}{\operatorname{Rc}(r)} \leq 1 / r \leq 2^{-7} \text {. }
\end{aligned}
$$

It follows from the above relations that

$$
2 L ! C_{1}^{L} r^{L} \leq e^{\left((3 / 2)+2^{-7}+2^{-4}+2^{-7}+2^{-3}+2^{-6}+2^{-7}+2^{-7}\right) c(r)} \leq e^{2 c(r)} .
$$

Substituting this inequality in (18), we obtain

$$
2 U<e^{2(L+1) c(r)} \prod_{i=1}^{k} \bar{x}_{i}^{n}{ }^{n} L_{0} \mid
$$

Similarly, we can also deduce that

$$
\begin{array}{r}
2(L+1) ! C_{1}^{L-1} C_{2} \leq r^{L R+2 R-(L+2)}\left(2 C^{2}\right)^{L(L+1)^{2} r^{2} /(2 R)} \\
\cdot(L+1)^{2[R+L(L+1)(g+1)+\sigma]+L+2+L(L+1) r / R} \cdot(4 H T C)^{(2 L+1) r} \\
\cdot(2 T)^{L[L(L+1)(g+1)+\sigma]} \cdot(2 H)^{(L+1) g[R+L(L+1)(g+1)+\sigma]} \\
\cdot(g+2)^{L R} \cdot[(g+2) r]^{L[L(L+1)(g+1)+\sigma]} \cdot e^{B} \\
\cdot r^{2[\sigma+L(L+1)(g+1)]+L+2} .
\end{array}
$$

Much as in the above calculations, we can also obtain the following inequalities:

$$
r^{(L+2)(R-1)}\left(2 C^{2}\right)^{L(L+1)^{2} r^{2} /(2 R)} \leq e^{(7 / 4) c(r)} \quad(\text { since } L \geq 1) ;
$$


$\frac{\{2[R+L(L+1)(g+1)+\sigma]+L+2+L(L+1) r / R\} \log (L+1)}{c(r)}$

$$
\begin{aligned}
& \leq \frac{2 R \log (L+1)}{c(r)}+\frac{\{2[L(L+1)(g+1)+\sigma]+L+2\} \log (L+1)}{c(r)}+\frac{L(L+1) r \log (L+1)}{R c(r)} \\
& \leq 2 / \log r+1 /(8 r)+3 /(4 r)+(\log r)^{\frac{2}{2}} /(8 r) \\
& \leq 2^{-6}+2^{-131}+2^{-128}+2^{-187} \leq 2^{-5} ; \\
& \quad \frac{(2 L+1) r \log (4 H C T)}{c(r)} \leq(L+1)^{-3} \leq 2^{-3} ; \\
& \quad \frac{L[L(L+1)(g+1)+\sigma] \log 2 T}{c(r)} \leq \frac{1}{2(L+1) r} \leq 2^{-7} ;
\end{aligned}
$$

$\frac{(L+1) g[R+L(L+1)(g+1)+\sigma] \log 2 H}{c(r)}$

$$
\begin{aligned}
& \leq \frac{\log B}{\log r}+\frac{1}{2(L+1)^{3} r}+\frac{1}{2(L+1) r} \leq 2^{-6}+2^{-132}+2^{-130}<2^{-5} \\
& \frac{L R \log (g+2)}{c(r)} \leq \frac{1}{\log r}+\frac{1}{2(L+1)^{3}} \leq 2^{-6}
\end{aligned}
$$

$\frac{L[L(L+1)(g+1)+\sigma] \log [(g+2) r]}{c(r)}$

$$
\begin{aligned}
& \quad \leq 1 /(2(L+1) r)+(\log r) /(2(L+1) r) \leq(\log r) / r<2^{-7} ; \\
& \frac{B}{C(r)} \leq \frac{B}{2(L+1)^{4} r \log B} \leq \frac{B}{2^{5} B^{64}}<2^{-7} ; \\
& \frac{\{2[\sigma+L(L+1)(g+1)]+L+2\} \log r}{C(r)} \leq \frac{\log r}{r}<2^{-7} .
\end{aligned}
$$

Substituting these inequalities in (19), we obtain

$$
2 V<e^{\left((7 / 4)+2^{-5}+2^{-3}+2^{-7}+2^{-5}+2^{-6}+2^{-7}+2^{-7}+2^{-7}-2\right) c(r)}<1 .
$$

From (17), (20) and (21), we deduce that

$$
\prod_{i=1}^{k} \bar{x}_{i}^{n_{i}}\left|L_{0}\right|>e^{-2(L+1) c(r)} \text {. }
$$

Since $b$ is any integer, it follows that

$$
\prod_{i=1}^{k} \bar{x}_{i} n_{i}\left\|_{i=1}^{k} \sum_{j=1}^{n_{i}} x_{i j} f_{i j}(\alpha)\right\|>e^{-2(L+1) c(r)} .
$$

Thus Theorem 1 is proved. 
The proof of Corollary 1.2 is quite similar to the proof of the corollary to Theorem 1 in [1]. Corollaries 1.2 and 1.3 plainly follow.

\section{Proof of Theorem 2}

From the hypotheses of Theorem 2 we know that the $f_{i}(z)$ $(i=1, \ldots, k)$ belong to an irreducible set of functions so $\sigma=q$. As in the proof of Theorem 1 , the integer $r$ must satisfy

$$
r \geq B^{4(k+1)^{4(g+q+1)^{2}}}+1
$$

namely, the inequality (4) holds. We shall use induction to prove the inequality (5).

Before commencing our induction, we introduce the following notation:

$$
\begin{aligned}
g_{\eta}(r) & =e^{-2 c_{\eta}(r)} r ! ; \\
c_{\eta}(r) & =(\eta+1)^{2}\left(g_{\eta}+q_{\eta}+1\right)\left(\log B_{\eta}\right)^{\frac{1}{2}} r(\log r)^{\frac{1}{2}} ; \\
H_{\eta} & =2(Z+2), \quad \eta=1,2, \ldots, k .
\end{aligned}
$$

In particular, $g=g_{k}, q=q_{k}, B=B_{k}, c(r)=c_{k}(r)$, $g(r)=g_{k}(r)$.

When $k=1$, it is clear that the inequality (5) follows from Corollary 1.2. We suppose $(5)$ is true for $k-1$ and prove it for $k$ $(k \geq 2)$. Put

$$
\begin{aligned}
\mu_{i} & =\left\|y f_{i}(\alpha)\right\|^{H_{k}(k+1)^{-1} c(x)}, i=1,2, \ldots, k, \\
\mu_{k+1} & =\left(\mu_{1} \ldots \mu_{k}\right)^{-1} .
\end{aligned}
$$

Clearly all $\mu_{i}>0, i=1, \ldots, k$. We consider two separate cases.

(i) There exists some $\mu_{j}(1 \leq j \leq k)$ satisfying $\mu_{j} \geq 1$. Without loss of generality, we assume $\mu_{k} \geq 1$. According to the induction hypotheses, the inequality (5) is true for $k-1$, namely 


$$
y\left\|y f_{1}(\alpha)\right\| \ldots\left\|y f_{k-1}(\alpha)\right\|>e^{-H_{k-1} c_{k-1}\left(r^{\prime}\right)}
$$

where $r^{\prime}$ is a positive integer satisfying the condition

$$
g_{k-1}\left(r^{\prime}\right) \leq y<g_{k-1}\left(r^{\prime}\right) \text {. }
$$

It is obvious that $g_{\eta} \leq g_{\eta+1}, q_{\eta} \leq q_{\eta+1}, B_{\eta} \leq B_{\eta+1} \quad(\eta=1, \ldots, k)$ from the definitions. Hence

$$
c_{k-1}(r)<(k+1)^{2}\left(g_{k}+q_{k}+1\right)\left(\log B_{k}\right)^{\frac{1}{2}} r(\log r)^{\frac{1}{2}}=c_{k}(r) .
$$

It follows that $g_{k-1}(r)>g_{k}(r)=g(r)$. This implies $r^{\prime}<r$. Because $c(r)$ is an increasing function of $r$, we have

$$
c_{k-1}\left(r^{\prime}\right)<c_{k-1}(r) \leq k^{2}(k+1)^{-2} c_{k}(r)
$$

Since $\mu_{k} \geq 1$ and

$$
k^{2}(k+1)^{-1} H_{k-1} / H_{k}+(k+1)^{-1}<1 \text { (when } k \geq 2 \text { ), }
$$

we obtain by (22) that

$$
\begin{aligned}
y\left\|y f_{1}(\alpha)\right\| \ldots\left\|y f_{k}(\alpha)\right\| & =y\left\|y f_{1}(\alpha)\right\| \ldots\left\|y f_{k-1}(\alpha)\right\| \mu_{k} \exp \left\{-H_{k}(k+1)^{-1} c(r)\right\} \\
& >\exp \left\{-k^{2}(k+1)^{-2} H_{k-1} c_{k}(r)-H_{k}(k+1)^{-1} c_{k}(r)\right\} \\
& =\exp \left\{-\left[k^{2}(k+1)^{-2} H_{k-1} / H_{k}+(k+1)^{-1}\right] H_{k} c_{k}(r)\right\} \\
& >\exp \left\{-H_{k} c_{k}(r)\right\}=e^{-2(k+2) c(r)} .
\end{aligned}
$$

Thus the inequality (5) is also true for $k$.

(ii) All the $\mu_{i}(i=1, \ldots, k)$ satisfy $0<\mu_{i}<1$. We suppose that the inequality (5) does not hold (when $k \geq 2$ ). Then there exists an integer $y \geq 2$ such that the following inequality holds:

$$
y\left\|y f_{1}(\alpha)\right\| \ldots\left\|y f_{k}(\alpha)\right\| \leq e^{-H_{k} c(r)} .
$$

Now let us consider a set of linear forms 


$$
\begin{aligned}
M_{i}(X) & =\mu_{i}^{-1}\left(x_{i}-f_{i}(\alpha) x_{k+1}\right), i=1, \ldots, k, \\
M_{k+1}(X) & =\mu_{k+1}^{-1} x_{k+1} .
\end{aligned}
$$

Denote by $v_{1}$ the first successive minimum of the parallelepiped defined by

$$
\left|M_{i}(X)\right| \leq 1, \quad 1 \leq i \leq k+1
$$

Further let $y_{1}, \ldots, y_{k}$ be a set of integers satisfying the following equalities :

$$
\left|y_{i}-y f_{i}(\alpha)\right|=\left\|y f_{i}(\alpha)\right\|, \quad i=1, \ldots, k \text {. }
$$

Since $y \geq 2,\left\{y, y_{1}, \ldots, y_{k}\right\}$ is a set of integers not all zero. By the definitions of $\mu_{i}$ 's and the inequality (23), we have

$$
\begin{aligned}
\left|\mu_{i}^{-1}\left(y_{i}-y f_{i}(\alpha)\right)\right| & =\mu_{i}^{-1}\left\|y f_{i}(\alpha)\right\|=e^{-H_{k}(k+1)^{-1} c(r)}, I \leq i \leq k, \\
\left|\mu_{k+1}^{-1} y\right| & =y\left\|y f_{1}(\alpha)\right\| \ldots\left\|y f_{k}(\alpha)\right\| e^{k(k+1)^{-1} H_{k} c(r)} \\
& \leq e^{-H_{k}(k+1)^{-1} c(r)} .
\end{aligned}
$$

According to the definition of successive minima, we have

$$
v_{1} \leq e^{-H_{k}(k+1)^{-1} c(r)}
$$

Let us consider the further set of linear forms

$$
\begin{aligned}
L_{i}(X) & =\mu_{i} x_{i}, \quad i=1, \ldots, k, \\
L_{k+1}(X) & =\mu_{k+1}\left(x_{1} f_{1}(\alpha)+\ldots+x_{k} f_{k}(\alpha)+x_{k+1}\right) .
\end{aligned}
$$

Without loss of generality, we can suppose that

$$
\mu_{1}^{-1}=\max \left(\mu_{1}^{-1}, \ldots, \mu_{k}^{-1}\right)>1 \text {. }
$$

Henceforth we suppose that $s$ is a positive integer such that 
$g(s-1) \leq \mu_{1}^{-1}<g(s)$. Likewise, $s$ must satisfy the following inequalities

$$
s \geq B^{4(k+1)^{4}(g+q+1)^{2}}+1
$$

and

$$
(s-1) ! \leq e^{2 c(r)} \mu_{1}^{-1}<s ! \text {. }
$$

Similarly, define the integers $s_{2}, \ldots, s_{k}$ by the inequalities

$$
\left(s_{i}^{-1) !} \leq e^{2 c(r)} \mu_{i}^{-1}<s_{i} !, \quad i=2, \ldots, k\right. \text {. }
$$

It is clear that $s=\max \left(s, s_{2}, \ldots, s_{k}\right)$. Finally, let

$$
R(s)=\left[(k+1) s(\log B / \log s)^{\frac{1}{2}}\right]+1 \text {. }
$$

Much as in the proof of Theorem 1, we can verify that the integers $s, s_{2}, \ldots, s_{k}$ and $R(s)$ satisfy all the hypotheses concerning $r, r_{1}, \ldots, r_{k}$ and $R$ in Lemmas 3,4 and 5 , respectively. Thus, according to Lemma 5, we can obtain $k+1$ linearly independent integer points

$$
\left(q_{i 0}, q_{i 1}, \ldots, q_{i k}\right), i=0,1, \ldots, k \text {, }
$$

such that, for $i=0,1, \ldots, k$,

$$
\left|q_{i j}\right| \leq c_{1} s_{j} ! \leq c_{1} s e^{2 c(s)} \mu_{j}^{-1}, j=0,1, \ldots, k \text {; }
$$

and

$$
\left|\sum_{j=0}^{k} q_{i j} f_{j}(\alpha)\right| \leq c_{2}\left(s ! s_{2} ! \cdots s_{k} !\right)^{-1} \leq c_{2} e^{-2 k c(s)} \mu_{k+1}^{-1},
$$

where $C_{1}$ and $C_{2}$ are the $(k+1)$ th successive minimum of the parallelepiped defined by

$$
\left|L_{i}(X)\right| \leq 1, i=1, \ldots, k+1 .
$$

Then $\lambda_{k+1}$ satisfies 
Linear forms in the values of E-functions

65

$$
\lambda_{k+1} \leq \max \left(C_{1} s e^{2 c(s)}, C_{2} e^{-2 k c(s)}\right)
$$

by (25). We shall prove that $C_{1} s e^{2 c(s)}>C_{2} e^{-2 k c(s)}$. As in the calculations in the proof of Theorem I, we can obtain the following inequalities:

$$
\begin{aligned}
\left(2 c^{2} H\right)^{(k+1) s} & <e^{(k+1)^{-3} c(s)} ; \\
(2 H)^{(k+t) g} & <e^{(k+1)^{-2} c(s)} ; \\
s^{2 t-1} & <e^{\left[(k+1)^{-2}+2 /(k+1)\right] c(s)} ; \\
(k+1)^{2 t+1} & <e^{\left[(k+1)^{-4}+2^{-50}(k+1)^{-2}\right] c(s)} .
\end{aligned}
$$

Thus we may deduce that

$$
\begin{aligned}
& \frac{c_{1} s e^{2 c(s)}}{c_{2} e^{-2 k c(s)}} \\
& =e^{2(k+1) c(s)} C_{1} s / C_{2} \\
& \geq e^{2(k+1) c(s)} \cdot\left(2 c^{2} H\right)^{-(k+1) s} \cdot(2 H)^{-(k+t) g} \cdot(k+1)^{-2 t-1} \cdot s^{-2 t+1} \\
& >\exp \left\{\left[2(k+1)-(k+1)^{-3}-2(k+1)^{-2}-2(k+1)^{-1}-(k+1)^{-4}-2^{-50}(k+1)^{-2}\right] c(s)\right\} \\
& >1 \text {. } \\
& \lambda_{k+1} \leq c_{1} s e^{2 c(s)} \text {. }
\end{aligned}
$$

We have, by Lemma 6 ,

$$
\nu_{1} \geq(k+1)^{-1} \lambda_{k+1}^{-1} \geq\left[(k+1) C_{1} s e^{2 c(s)}\right]^{-1} \text {. }
$$

As in the proof of Theorem 1, we have

$$
\begin{aligned}
& \left(2 c^{2}\right)^{(k+1)^{2} s^{2} /(2 R(s))} s^{R(s)-1} \leq e^{(3 / 2)(k+1)^{-1} c(s),} \\
& {[(g+2) T]^{R(s)+k(k+1)(g+1)+q+1} \leq e^{2^{-2}(k+1)^{-1} c(s),}}
\end{aligned}
$$

https://doi.org/10.1017/S0004972700005037 Published online by Cambridge University Press 


$$
\begin{aligned}
(2 H)^{s+g[R(s)+k(k+1)(g+1)+q]} & \leq e^{2^{-3}(k+1)^{-1} c(s),} \\
s^{k(k+1)(g+1)+q+2} & \leq e^{2^{-4}(k+1)^{-1} c(s)}, \\
(k+1)^{(k+1) s / R(s)+1} & \leq e^{2^{-4}(k+1)^{-1} c(s)} .
\end{aligned}
$$

We can deduce from the above relations that

$$
\begin{aligned}
(k+1) C_{1} s e^{2 c(s)} \leq & e^{2 c(s)} \cdot\left(2 c^{2}\right)^{(k+1)^{2} s^{2} /(2 R(s)]_{s} R(s)-1} \\
& \cdot[(g+2) T]^{R(s)+k(k+1)(g+1)+q+1} \cdot(2 H)^{s+g[R(s)+k(k+1)(g+1)+q]} \\
& \cdot s^{k(k+1)(g+1)+q+2} \cdot(k+1)(k+1) s / R(s)+1 \\
& <e^{2 c(s)} \cdot \exp \left\{\left((3 / 2)+2^{-2}+2^{-3}+2^{-4}+2^{-4}\right)(k+1)^{-1} c(s)\right\} \\
& =e^{\left(2+2(k+1)^{-1}\right) c(s)}=e^{H_{k}(k+1)^{-1} c(s)} .
\end{aligned}
$$

Hence

$$
v_{1}>e^{-H_{k}(k+1)^{-1} c(s)}
$$

We shall show that

$$
c(s) \leq c(r) .
$$

By the definition of $\mu_{1}$, we have

$$
y \mu_{1}=y\left\|y f_{1}(\alpha)\right\| e^{H_{k}(k+1)^{-1} c(r)} \text {. }
$$

By the definition of $c_{1}(r)$, we see that

$$
\begin{aligned}
c_{1}(r) & =2^{2}\left(g_{1}+q_{1}+1\right)\left(\log B_{1}\right)^{\frac{1}{2}} r(\log r)^{\frac{1}{2}} \\
& \leq 4(k+1)^{-2} c(r)<c(r) \quad(k \geq 2) ;
\end{aligned}
$$

hence

$$
g_{1}(r)=e^{-2 c_{1}(r)} r !>e^{-2 c(r)} r !=g(r) .
$$

Denote by $r^{\prime \prime}$ a positive integer satisfying 


$$
g_{1}\left(r^{\prime \prime}-1\right) \leq y<g_{1}\left(r^{\prime \prime}\right)
$$

Since $g_{1}(r)>g(r)$, it follows that $r^{\prime \prime}<r$. This implies

$$
c_{1}\left(r^{\prime \prime}\right) \leq c_{1}(r) \leq 4(k+1)^{-2} c(r)
$$

By the conclusion of Corollary $1.2(k=1)$ and (27), we obtain

$$
\begin{aligned}
y \mu_{1 .} & =y\left\|y f_{1}(\alpha)\right\| e^{H_{k}(k+1)^{-1} c(x)}>e^{-4 c_{1}\left(r^{\prime \prime}\right)+H_{k}(k+1)^{-1} c(r)} \\
& \geq \exp \left\{\left[-16(k+1)^{-2}+2(k+2)(k+1)^{-1}\right] c(r)\right\}>1 \quad(k \geq 2),
\end{aligned}
$$

hence $\mu_{1}^{-1}<y$. It follows that $s \leq r$ by the definitions of $s$ and $r$. Thus (26) is true. Finally, we obtain

$$
\nu_{1}>e^{-H_{k}(k+1)^{-1} c(r)} \text {. }
$$

This is contrary to inequality (24), hence the assumption (23) is not valid. This proves Theorem 2 .

The proof of Corollary 1.2 is quite similar to the proof of the corollary to Theorem 2 of [1].

\section{Remarks}

If $\mathbb{K}$ were an imaginary quadratic field $(\mathbb{K}=Q(\sqrt{-d}))$ in Theorem I and its corollaries, then we could use Lemma 31 of Schneider [4] in place of Lemma 1 here to construct the auxiliary polynomials in Lemma 2. Further we note that the conjugate to $\beta(B \in \mathbb{K})$ is its complex conjugate $\bar{\beta}$, so $|B|=|\bar{B}|$. Thus all the details of the proofs of the theorems and corollaries are as in the case $\mathbb{K}=Q$. Only the parameter $B$ depends on $d$. Therefore, if $\mathbb{K}$ were an imaginary quadratic field, we would also obtain Theorem 1 and its corollaries.

\section{References}

[1] Kurt Mahler, "On a paper by A. Baker on the approximation of rational powers of $e$ ", Acta Arith. 27 (1975), 61-87. 
[2] Kurt Mahler, Lectures on transcendental numbers (Lecture Notes in Mathematics, 546. Springer-Verlag, Berlin, Heidelberg, New York, 1976).

[3] Ю.H. Манаров [Yu.N. Makarov], "Об оценнах меры лннейной незавнсимости значений Е-фаннций" [On the estimates of the measure of linear independence for the values of E-functions], Vestnik Moskov. Univ. Ser. I Mat. Meh. 2 (1978), 3-12.

[4] Theodor Schneider, Einführung in die Transzendenten Zahlen (Die Grundlehren der Mathematischen Wissenschaften, 81. SpringerVerlag, Berlin, Göttingen, Heidelberg, 1957).

[5] А.Б. Шияловсний [A.В. Šidlovski ], "О трансцендентности н алгебраичесной незавнснмости значений целых фуннций неноторых нлассов" [Transcendence and algebraic independence of the values of entire functions of certain classes], Moskov. Gos. Univ. Ǔ́. Zap. 186 (1959), 11-70.

[6] А.Б. Шидловсний [A.В. ŠidlovskiT], "О нримерни алгебраичесной независимостн значений одного нласса целых фуннщнй" [A criterion for algebraic independence of the values of a class of entire functions], Izv. Akad. Nauk SSSR Ser. Mat. 23 (1959), 35-66; Amer. Math. Soc. Trans l. (2) 22 (1962), 339-370.

[7] A.B. Shidlovskii, "On the estimates of the algebraic independence measures of the values of E-functions", J. Austral. Math. Soc. Ser. A 27 (1979), 385-407.

[8] Keijo väänänen, "On lower estimates for linear forms involving certain transcendental numbers", Bull. Austral. Math. Soc. 14 (1976), 161-179.

[9] Keijo Väänänen, "On linear forms of the values of one class of E-functions", Acta Univ. Oulu. Ser. A Sci. Rerum Natur. No. 41 Math. No. 12 (1976), 19pp.

[10] Xu Guang Shan and Yu Kun Rui, "Some diophantine inequalities involving a class of Siegel's E-functions", Kexue Tongbao 24 (1979), 481-486. 
[11] Yu Kun Rui and Xu Guang Shan, "A note on a theorem of Baker and Mahler", Acta Math. Sinica 22 (1979), 487-494.

School of Mathematics and Physics,

Macquarie University,

North Ryde,

New South Wales 2113,

Australia.

Permanent Address:

Institute of Mathematics,

Academia Sinica,

Beijing,

China. 